\title{
Tuning the Two-Dimensional Electron Gas at Oxide Interfaces with Ti-O Configurations: Evidence from X-ray Photoelectron Spectroscopy
}

Zhang, Yu ; Gan, Yulin; Niu, Wei ; Norrman, Kion; Yan, Xi ; Christensen, Dennis Valbjørn; von Soosten, Merlin; Zhang, Hongrui ; Shen, Baogen; Pryds, Nini

Total number of authors:

12

Published in:

A C S Applied Materials and Interfaces

Link to article, DOI:

10.1021/acsami.7b16510

Publication date:

2018

Document Version

Peer reviewed version

Link back to DTU Orbit

Citation (APA):

Zhang, Y., Gan, Y., Niu, W., Norrman, K., Yan, X., Christensen, D. V., von Soosten, M., Zhang, H., Shen, B. Pryds, N., Sun, J., \& Chen, Y. (2018). Tuning the Two-Dimensional Electron Gas at Oxide Interfaces with Ti-O Configurations: Evidence from X-ray Photoelectron Spectroscopy. A C S Applied Materials and Interfaces, 10(1), 1434-1439. https://doi.org/10.1021/acsami.7b16510

\section{General rights}

Copyright and moral rights for the publications made accessible in the public portal are retained by the authors and/or other copyright owners and it is a condition of accessing publications that users recognise and abide by the legal requirements associated with these rights.

- Users may download and print one copy of any publication from the public portal for the purpose of private study or research.

- You may not further distribute the material or use it for any profit-making activity or commercial gain

- You may freely distribute the URL identifying the publication in the public portal 


\title{
Tuning the Two-Dimensional Electron Gas at Oxide
}

\section{Interfaces with Ti-O Configurations: Evidence from X-}

\section{ray Photoelectron Spectroscopy}

Yu Zhang ${ }^{1,2}$, Yulin Gan ${ }^{2}$,Wei Niu' ${ }^{2}$, Kion Norrman ${ }^{2}$, Xi Yan ${ }^{1}$, Dennis Valbjørn Christensen ${ }^{2}$, Merlin von Soosten ${ }^{2}$, Hongrui Zhang ${ }^{1}$, Baogen Shen ${ }^{1}$, Nini Pryds ${ }^{2}$, Jirong Sun ${ }^{1 *}$ and Yunzhong Chen ${ }^{2 *}$

${ }^{1}$ Beijing National Laboratory for Condensed Matter Physics and Institute of Physics, Chinese Academy of Sciences, Beijing 100190, Peoples' Republic of China

School of Physical Sciences, University of Chinese Academy of Sciences, Beijing 100049, Peoples'

Republic of China

${ }^{2}$ Department of Energy Conversion and Storage, Technical University of Denmark, Risø Campus, 4000 Roskilde, Denmark

KEYWORDS: two-dimensional electron gas, redox reaction, $\mathrm{TiO}_{2}, \mathrm{SrTiO}_{3}$, oxygen vacancies.

\begin{abstract}
Chemical redox reaction can lead to a two-dimensional electron gas (2DEG) at the interface between a $\mathrm{TiO}_{2}$-terminated $\mathrm{SrTiO}_{3}(\mathrm{STO})$ substrate and an amorphous $\mathrm{LaAlO}_{3}(\mathrm{a}-\mathrm{LAO})$ capping layer. When replacing the STO substrate with rutile and anatase $\mathrm{TiO}_{2}$ substrates, considerable differences in interfacial conduction are observed. Based on X-ray photoelectron spectroscopy (XPS) and transport measurements, we conclude that the interfacial conduction comes from redox reactions,
\end{abstract}


and that the differences among the materials systems result mainly from variations in the activation energies for the diffusion of oxygen vacancies at substrate surfaces.

\section{INTRODUCTION}

As silicon is the foundation material for semiconductor technology, strontium titanate, $\mathrm{SrTiO}_{3}$ (STO), is the base material for the emerging field of oxide electronics. Two-dimensional electron gases (2DEGs) at STO-based heterointerfaces, ${ }^{1-4} \mathrm{LaAlO}_{3} / \mathrm{SrTiO}_{3}(\mathrm{LAO} / \mathrm{STO})$ in particular, ${ }^{1}$ exhibit a large number of remarkable physical properties, such as superconductivity, ${ }^{5}$ magnetism, ${ }^{6}$ sensitivity to electric field, ${ }^{7}$ light illumination, ${ }^{8}$ and high electron mobility, ${ }^{9-10}$ which show potential applications in the next generation of electronic devices. ${ }^{11}$ Despite intensive research, the origin of the conductivity at the LAO/STO interface remains hotly debated. ${ }^{12-17}$ Different scenarios have been proposed, including the charge transfer from LAO surface to interface due to polar discontinuity, ${ }^{1,12}$ extrinsic doping by generation of oxygen vacancies in STO substrate, ${ }^{4,13-15}$ and extrinsic doping by cation intermixing across the interface to induce $n$-type conduction of La-doped STO. ${ }^{16}$ Among these, formation of oxygen vacancies into STO substrate frequently occurs while depositing oxide capping layers ${ }^{18-24}$ that contain specific active elements, such as Al, Hf, Zr and Ti on the top of STO. During the deposition process, oxygen ions in the STO substrate will diffuse outward to oxidize the reactive plasma species absorbed on the substrate surface, leaving behind oxygen vacancies in the STO substrate. ${ }^{18}$ This in turn gives rise to a 2DEG confined to the interface as what occurred for the amorphous- $\mathrm{LaAlO}_{3}$ (aLAO)/STO interface grown at room temperature. ${ }^{18}$ Using $\mathrm{O}^{18}$-exchanged STO substrates, the significant oxygen transfer from substrate to the film has been experimentally observed for both high

temperature and room temperature film deposition using pulsed laser deposition (PLD). ${ }^{25-26}$ Agham et 
$a l^{21}$ also reported that when metals were deposited on the STO substrate, an oxygen-deficient layer in STO is very likely to be created if the formation energy of the metal oxide is larger than that of an oxygen vacancy. So far, generating oxide 2DEGs by redox reactions has been largely limited to the STO substrate, for which various capping layers have been adopted, such as a-LAO, ${ }^{22}$ amorphous $\mathrm{CaHfO}_{3},{ }^{20}$ yttria-stabilized zirconia (YSZ),${ }^{18}$ and $\mathrm{Al}_{2} \mathrm{O}_{3} .{ }^{4,19}$ Besides (001)-oriented STO substrates, STO with orientations of (110) and (111) $)^{27}$ has also been used for the deposition of a-LAO. Recently, Sarkar $e t a l^{28}$ replaced the $\mathrm{STO}$ substrate of a-LAO/STO heterostructures with anatase $\mathrm{TiO}_{2}\left[\mathrm{TiO}_{2}(\mathrm{~A})\right]$ and rutile $\mathrm{TiO}_{2}\left[\mathrm{TiO}_{2}(\mathrm{R})\right]$ and obtained interfacial $2 \mathrm{DEGs}$ with different transport properties from aLAO/STO. However, the origin underlying the transport differences remains open. Herein, we employed both transport and X-ray photoelectron spectroscopy (XPS) measurements to study the formation of $2 \mathrm{DEG}$ s between a-LAO and $\mathrm{STO}, \mathrm{TiO}_{2}(\mathrm{~A})$ or $\mathrm{TiO}_{2}(\mathrm{R})$ with different Ti-O-Ti bond configurations. Similar to previous reports, we found that the a-LAO/STO interface is metallic from $295 \mathrm{~K}$ to $2 \mathrm{~K}$ with a typical carrier density of $1.0 \times 10^{14} \mathrm{~cm}^{-2}$ at $295 \mathrm{~K}$. The a-LAO/TiO $2(\mathrm{~A})$ interface shows a similar metallic behavior to a-LAO/STO except for a resistance upturn below $125 \mathrm{~K}$, which is likely to be due to the Kondo effect. Moreover, the a- $\mathrm{LAO} / \mathrm{TiO}_{2}(\mathrm{R})$ interface exhibits a carrier density lower by a factor of 18 than the former two systems and displays a metal-to-insulator transition at $45 \mathrm{~K}$ upon cooling. Our XPS measurements indicate that the differences in transport properties of these three heterostructures is ascribed mainly to the different activation energies for the diffusion of oxygen vacancies from the substrate to the a-LAO capping layer during the interfacial redox reactions.

\section{EXPERIMENTAL DETAILS}

Three types of substrates were employed, $\mathrm{STO}(001), \mathrm{TiO}_{2}(\mathrm{~A})(001)$ and $\mathrm{TiO}_{2}(\mathrm{R})(001)$ with a size of $5 \times 5 \times 0.5 \mathrm{~mm}^{3}$. The $\mathrm{TiO}_{2}(\mathrm{R})$ and $\mathrm{STO}$ are commercial substrates. To get a $\mathrm{TiO}_{2}$-terminated layer, 
the STO substrate was chemically etched by a $\mathrm{HCl}-\mathrm{HNO}_{3}$ acidic solution, and then annealed at $1000{ }^{\circ} \mathrm{C}$ for $1 \mathrm{~h}$ in 1 bar oxygen. The crystalline $\mathrm{TiO}_{2}(\mathrm{~A})$ substrate was grown by PLD (KrF excimer laser, $\lambda=248 \mathrm{~nm}$ ) on the LAO substrate at $750^{\circ} \mathrm{C}$ under an oxygen pressure of $5 \times 10^{-3} \mathrm{mbar}$. The films obtained (about $100 \mathrm{~nm}$ ) are confirmed to be of high crystalline quality by X-ray diffraction (XRD), with a smooth surface (root mean square roughness is $1.1 \mathrm{~nm}$, Supporting Information S1). The heterostructures were formed by capping the three substrates with a-LAO films grown by PLD with a commercial LAO single crystal target. This amorphous capping layer is nonpolar therefore rules out the polar discontinuity induced electronic reconstruction in the all-crystalline heterointerface. ${ }^{1,29}$ During the deposition, the substrate was kept at room temperature and the oxygen pressure was maintained to $10^{-6} \mathrm{mbar}$. The laser fluence is $3 \mathrm{Jcm}^{-2}$ and the repetition rate is $1 \mathrm{~Hz}$. The target-substrate distance was fixed at $5 \mathrm{~cm}$. The thickness of the a-LAO capping layer was kept at $3 \mathrm{~nm}$ in this work. However, samples with the a-LAO films up to the thickness of $16 \mathrm{~nm}$ showed similar results (Supporting Information S4).

Transport measurements were done in both van der Pauw and Hall-bar geometry with the latter prepared by depositing a-LAO through a mechanical mask. Ultrasonic Al wire bonding was used to get electric connection. The electric measurements were performed in a CRYOGENIC cryogen-free measurement system in the temperature range from $300 \mathrm{~K}$ to $2 \mathrm{~K}$ and magnetic field ranges from $0 \mathrm{~T}$ to $16 \mathrm{~T}$.

The valence state of relevant elements after the deposition of a-LAO was investigated by a Thermo Scientific ESCALAB Xi ${ }^{+}$XPS, using a monochromatic Al Ka X-ray source with a photon energy of $1,486.6 \mathrm{eV}$. This leads to a kinetic energy of Ti $2 p$ electrons of roughly $1,028 \mathrm{eV}$. The pass energy used for the high resolution scan was $50 \mathrm{eV}$. For analyzing the Ti $2 p_{3 / 2}$ peaks, a Smart 
background was subtracted and the spectra were normalized to the total area below the Ti peaks $\left([\mathrm{Ti}]=\left[\mathrm{Ti}^{4+}\right]+\left[\mathrm{Ti}^{3+}\right]+\left[\mathrm{Ti}^{2+}\right]=100 \%\right)$. The binding energies obtained from this work $(458.6 \pm 0.1 \mathrm{eV}$ for $\mathrm{Ti}^{4+}, 457.0 \pm 0.1 \mathrm{eV}$ for $\mathrm{Ti}^{3+}$, and $455.3 \pm 0.1 \mathrm{eV}$ for $\mathrm{Ti}^{2+}$ ) are in excellent agreement with the corresponding values $(458.7 \pm 0.2 \mathrm{eV}, 457.1 \pm 0.4 \mathrm{eV}$, and $455.3 \pm 0.4 \mathrm{eV})$ from the NIST Standard Reference Database 20, Version 4.1 (web version).

\section{RESULTS}

Fig. 1(a) is a schematic illustration of the heterostructure investigated here which consists of an aLAO capping layer deposited on one of the three $\mathrm{TiO}_{2}$-based substrates: $\mathrm{STO}, \mathrm{TiO}_{2}(\mathrm{~A})$ and $\mathrm{TiO}_{2}(\mathrm{R})$. To illustrate the intrinsic difference in the Ti-O configuration among the three substrates, the atomic structures of the three (001)-orientated substrates are also shown in Fig. 1(b)-(d). The STO substrate is

a perovskite oxide with a lattice parameter of $3.905 \AA$, which consists of alternating uncharged $\mathrm{SrO}$ and $\mathrm{TiO}_{2}$ sublayers. Its $\mathrm{TiO}_{2}$-terminated surface has a Ti-O-Ti angle as large as $180^{\circ}$. The $\mathrm{TiO}_{2}(\mathrm{~A})$ and $\mathrm{TiO}_{2}(\mathrm{R})$ belong to the ditetragonal dipyramidal class with different lattice parameters: $\mathrm{a}=\mathrm{b}=3.784 \AA$, $\mathrm{c}=9.515 \AA$ for the $\mathrm{TiO}_{2}(\mathrm{~A})$ and $\mathrm{a}=\mathrm{b}=4.594 \AA, \mathrm{c}=2.959 \AA$ for the $\mathrm{TiO}_{2}(\mathrm{R})$. The Ti-O-Ti angle is $155.4^{\circ}$ for the $\mathrm{TiO}_{2}(\mathrm{~A})$ and $130.4^{\circ}$ and $99.1^{\circ}$ for the $\mathrm{TiO}_{2}(\mathrm{R})$. Shortly, by changing the substrate from STO to $\mathrm{TiO}_{2}(\mathrm{~A})$ and then to $\mathrm{TiO}_{2}(\mathrm{R})$, we can continuously decrease the Ti-O-Ti angle, thus tune the hybridization between $\mathrm{O}_{2 p}$ and $\mathrm{Ti}_{3 d}$ e orbitals. ${ }^{30}$

The temperature-dependent sheet resistance $\left(R_{\mathrm{s}}\right)$, sheet carrier density $\left(n_{\mathrm{s}}\right)$ and mobility $(\mu)$ of the three heterostructures are shown in Fig. 2. The a-LAO/STO heterointerface is metallic as reported before. ${ }^{18,22}$ The Hall resistance, $R_{\mathrm{xy}}$, is nonlinear (Fig. 3(a)) below $50 \mathrm{~K}$ which is probably due to the presence of two-band transport carriers (details on the two-band model are shown in the Supporting 
Information S2). ${ }^{31}$ The $n_{\mathrm{s}}$ in Fig. 2(b) is deduced from the linear part of $R_{\mathrm{xy}}$ in the low magnetic field in Fig. 3(a) and it is nearly constant in the temperature $(T)$ range of $100-295 \mathrm{~K}$ with a value of $1.0 \times 10^{14}$ $\mathrm{cm}^{-2}$. As $T$ decreases below $100 \mathrm{~K}$, the $n_{\mathrm{s}}$ decreases and reaches a value around $2.2 \times 10^{13} \mathrm{~cm}^{-2}$ at $2 \mathrm{~K}$ due to the carrier freeze-out. The $\mu$ increases upon cooling and is about $370 \mathrm{~cm}^{2} \mathrm{~V}^{-1} \mathrm{~s}^{-1}$ at $2 \mathrm{~K}$.

The a-LAO/TiO 2 (A) heterointerface shows a metallic behavior similar to the a-LAO/STO in the temperature range $125 \mathrm{~K}<T<295 \mathrm{~K}$. As the temperature decreases below $125 \mathrm{~K}$, the $R_{\mathrm{S}}$ for the a$\mathrm{LAO} / \mathrm{TiO}_{2}(\mathrm{~A})$ displays a smooth upturn. Two possibilities account for this upturn: (1) a Kondo effect ${ }^{32-}$ ${ }^{33}$ caused by the enhanced spin scattering of charge carriers by localized magnetic moments, ${ }^{34-35}$ or (2) a 2D weak localization effect, caused by self-interference of wave packets when they are coherently back scattered by impurities. ${ }^{36}$ In order to distinguish between these two effects, we implement the Kondo effect mode ${ }^{37}$ as well as $2 \mathrm{D}$ weak localization mode ${ }^{38}$ to study what is the origin of the resistance upturn. Compared to the weak localization, the Kondo effect can better explain our $R_{\mathrm{S}}-T$ behavior, which is in contrast to the mechanism described by Sarkar et al. ${ }^{28}$ The Kondo model was fitted using the following expression ${ }^{37}$ :

$$
R(T)=R_{0}+\mathrm{q} T^{2}+\mathrm{p} T^{5}+R_{K, 0}\left(\frac{1}{1+\left(2^{1 / s-1)\left(T / T_{\mathrm{K}}\right)^{2}}\right.}\right)^{\mathrm{s}}
$$

where $R_{0}$ is the residual resistance due to the disorder, the $T^{2}$ and $T^{5}$ terms represent the temperature dependences of electron-electron and electron-phonon interactions, respectively. The last term in Eqs. (1) is the Kondo contribution, where $R_{\mathrm{K}, 0}$ is the Kondo resistance at zero temperature and $T_{\mathrm{K}}$ is the Kondo temperature defined as the temperature at which the Kondo resistance is half relative to its zero-temperature value. The parameter $\mathrm{s}$ is fixed at 0.225 according to the theoretical result obtained from the numerical renormalization group. ${ }^{39-40}$ As shown in Fig. 
3(b), the $R_{\mathrm{S}}$ was well fitted and the relative coefficients are: $R_{0}=942.6 \Omega, \mathrm{q}=7.3 \times 10^{-4} \Omega / \mathrm{K}^{2}, \mathrm{p}=$ $1.3 \times 10^{-9} \Omega / \mathrm{K}^{5}, R_{\mathrm{K}, 0}=1202.9 \Omega$ and $T_{\mathrm{K}}=86.2 \mathrm{~K}$. When $T<<T_{\mathrm{K}}$, the Kondo singlet state ${ }^{41}$ emerges and the magnetic scattering centers are screened by charge carriers and turn nonmagnetic. This effect is manifested itself by the $R_{\mathrm{s}}$ upturn which slows down and saturates at low temperatures. ${ }^{37}$ In contrast, for the 2D weak localization effect, the $R_{\mathrm{s}}$ upturn will never saturate (Supporting Information S3). As for the $n_{\mathrm{s}}$ of the a-LAO/TiO $2(\mathrm{~A})$, it is nearly constant, $(1.3-2.1) \times 10^{14} \mathrm{~cm}^{-2}$, from $295 \mathrm{~K}$ to $2 \mathrm{~K}$, which is comparable to that of the a-LAO/STO for $T>125 \mathrm{~K}$. Meanwhile, the $\mu$ of a-LAO/TiO 2 (A) locates at the range from 13.0 to $24.5 \mathrm{~cm}^{-2} \mathrm{~V}^{-1} \mathrm{~s}^{-1}$ from $295 \mathrm{~K}$ to $2 \mathrm{~K}$, much lower than that of a-LAO/STO for $T<125 \mathrm{~K}$. The low electron mobility of the a-LAO/TiO 2 (A) could be due to the Kondo scattering.

As the temperature decreases, the a- $\mathrm{LAO} / \mathrm{TiO}_{2}(\mathrm{R})$ first exhibits a metallic-like behavior from 295 $\mathrm{K}$ to $45 \mathrm{~K}$ and then a metal-to-insulator transition around $45 \mathrm{~K}$, due to the freeze-out of charge carriers. For $T<20 \mathrm{~K}, R_{\mathrm{s}}$ becomes so large that it is well beyond the limits of our measurement system (Fig. 2(a)). Hall effect measurements are therefore only reliable in the temperature range of $30 \mathrm{~K}<T<125$ $\mathrm{K}$, which display a $n_{\mathrm{s}}$ of $5.6 \times 10^{12} \mathrm{~cm}^{-2}$ at $125 \mathrm{~K}$ and $2.5 \times 10^{11} \mathrm{~cm}^{-2}$ at $30 \mathrm{~K}$ (Fig. 2(b)). The value of $n_{\mathrm{s}}$ for the a-LAO/ $\mathrm{TiO}_{2}(\mathrm{R})$ is more than 2 orders of magnitude lower than the former two systems at low temperature $(T \leq 30 \mathrm{~K})$. In contrast, $\mu$ increases as temperature decreases from $125 \mathrm{~K}$ to $30 \mathrm{~K}$ and reaches a value of $77 \mathrm{~cm}^{-2} \mathrm{~V}^{-1} \mathrm{~s}^{-1}$ at $30 \mathrm{~K}$ (Fig. 2(c)). It is comparable with that of a-LAO/STO, and higher than that of a-LAO/TiO $2(\mathrm{~A})$. Notably, below $38 \mathrm{~K}$, the $R_{\mathrm{S}}$ can be well fitted by a small polaron $\operatorname{model}^{42}$ (Fig. 3(c)):

$$
\ln \left(\frac{R_{\mathrm{s}}}{T}\right)=\ln (\mathrm{A})+\frac{E_{\mathrm{p}}}{k_{\mathrm{B}} T}
$$


where $\mathrm{A}$ is a temperature independent constant, $E_{\mathrm{p}}$ is the activation energy for hopping condution and $k_{\mathrm{B}}$ is the Boltzmann constant. Through fitting, the deduced activation energy is $14 \mathrm{meV}$ that is comparable with Co doped $\mathrm{TiO}_{2},{ }^{43}$ indicating that the transport proceeds through thermal activation. ${ }^{44}$

To reveal the physical origin of the interfacial conductivity and mechanisms that lead to the different transport properties of these three heterostructures, we further performed an analysis of the oxidization state of relevant elements by XPS. In the stoichiometric single crystalline $\mathrm{STO}$ and $\mathrm{TiO}_{2}$ substrates, the valence states of the $\mathrm{Ti}$ cations and $\mathrm{O}$ anions are $\mathrm{Ti}^{4+}$ and $\mathrm{O}^{2-}$, respectively. During the deposition of a-LAO, the $\mathrm{O}$ anions will diffuse out of the substrate to oxidize the reactive plasma species absorbed on the substrate surface. ${ }^{18,21}$ This results in the formation of oxygen vacancies. Obviously, once an oxygen vacancy forms, two electrons will be transferred to nearby $\mathrm{Ti}^{4+}$ cations, reducing the valence state of $\mathrm{Ti}^{4+}$ cations to either $\mathrm{Ti}^{3+}$ or $\mathrm{Ti}^{2+}$. Fig. 4(a) shows the $\mathrm{Ti} 2 p$ spectra in the three heterostructures. The main peaks at the binding energy of $458.6 \mathrm{eV}$ correspond to $\mathrm{Ti}^{4+}$ cations, and the right side shoulders at the binding energy of $457 \mathrm{eV}$ and $455.3 \mathrm{eV}$ stem from $\mathrm{Ti}^{3+}$ and $\mathrm{Ti}^{2+}$ cations. A quantitative analysis shown in Fig. 4(b)-(d) gives the relative concentrations of $\mathrm{Ti}^{3+}$ and $\mathrm{Ti}^{2+}$ cations (Table I). It turns out, among these three heterostructures, the a-LAO/ $\mathrm{TiO}_{2}(\mathrm{~A})$ has the largest percentages of $\mathrm{Ti}^{3+}$ and $\mathrm{Ti}^{2+}$ cations, which are $17.5 \%$ and $6.5 \%$, respectively. The a-LAO/STO has comparable percentages of $\mathrm{Ti}^{3+}$ and $\mathrm{Ti}^{2+}$ cations (15.9\% and 4.7\%), whereas a-LAO/TiO $2(\mathrm{R})$ shows the lowest $\mathrm{Ti}^{3+}$ and $\mathrm{Ti}^{2+}$ concentrations (5.7\% and 1.8\%) more than three times lower than the former two systems. Shortly, the $\mathrm{Ti}^{3+}$ and $\mathrm{Ti}^{2+}$ concentrations and thus the concentration of oxygen vacancies in the $\mathrm{TiO}_{2}$-based substrates show a similar trend with $n_{\mathrm{s}}$ upon changing the substrates from $\mathrm{STO}$ to $\mathrm{TiO}_{2}(\mathrm{~A})$, and then to $\mathrm{TiO}_{2}(\mathrm{R})$. Note that the carrier concentration deduced from XPS is always higher than that of $n_{\mathrm{s}}$ determined by Hall measurements. This could be due to the presence of localized carriers which 
can be detected by XPS but not sensitive to Hall measurements. Based on the XPS result and the nonpolar of capping layers, we can conclude that the mobile charge carriers in the heterointerfaces mainly come from the oxygen vacancies in the substrates. It should be noted that although there are reports on that $\mathrm{Ti}$ interstitials contribute to the band gap states ${ }^{45}$ or the $\mathrm{Ti}^{3+}$ and $\mathrm{Ti}^{2+}$ concentrations of $\mathrm{TiO}_{2}(\mathrm{R})$ surface. However, recent electron bombardment results confirm that oxygen vacancies rather than Ti interstitials make the dominant contribution to the electron reconstruction. ${ }^{46}$

\section{DISCUSSION}

The difference in transport properties of these three types of conductive heterointerfaces is related to the different concentrations of oxygen vacancies in the substrates. Since we used the same capping layer of a-LAO, therefore, the driving force underlying the redox reaction remains the same. The different content of oxygen vacancies should result from a different surface formation energy of oxygen vacancy (the enthalpy required to form one surface oxygen vacancy) and/or the different activation energy for the diffusion of oxygen vacancy (the energy barrier for an oxygen vacancy migration from one position to another at the substrate surface). According to the previous calculations as summarized in Table II, $\mathrm{TiO}_{2}$-terminated STO substrate has the highest surface formation energy of oxygen vacancy among the three substrates, which is $5.94 \mathrm{eV},{ }^{47} \mathrm{TiO}_{2}(\mathrm{~A})$ has a medium value in the range of $4.00-4.96 \mathrm{eV},{ }^{48}$ and $\mathrm{TiO}_{2}(\mathrm{R})$ possesses the lowest formation energy of oxygen vacancy on its surface $\left(3.21 \mathrm{eV}^{49}\right)$. These oxygen vacancy formation energies seem to be monotonically increasing with the increase of Ti-O-Ti angles, such as that STO has the largest Ti-O-Ti angle $\left(180^{\circ}\right)$ and also exhibits the highest oxygen vacancy formation energy. Therefore, under the same deposition condition, oxygen vacancies are easiest to be formed in $\mathrm{TiO}_{2}(\mathrm{R})$, then in $\mathrm{TiO}_{2}(\mathrm{~A})$, and finally in STO. However, the a-LAO/ $\mathrm{TiO}_{2}(\mathrm{R})$ shows the lowest carrier concentration as well as oxygen 
vacancies. This indicates that the difference in the surface formation energy of oxygen vacancy plays a negligible role in the measured difference. In other words, the driving force of the a-LAO deposition can overcome all the barriers for the interface redox reaction. Instead, the less carrier/oxygen vacancy concentration of a-LAO/ $\mathrm{TiO}_{2}(\mathrm{R})$ is more likely due to the rather large activation energy for the diffusion of oxygen vacancies at the $\mathrm{TiO}_{2}(\mathrm{R})$ surface, which is around $1.5 \mathrm{eV}$ due to the small Ti-O-Ti bond angle. In contrast, the activation energy for the diffusion of oxygen vacancy at the surface of STO and $\mathrm{TiO}_{2}(\mathrm{~A})$ is much smaller, which could be only $0.1-0.2 \mathrm{eV} .47,50$ Therefore, it is the activation dynamics of oxygen vacancies that determine the differences in the formation of 2DEGs among these three heterostructures. Since the activation energy for the diffusion of oxyen vacancies is high at the $\mathrm{TiO}_{2}(\mathrm{R})$ surface, the formation of oxygen vacancy is probably thermodynamically limited to the substrate surface. Compared to the other two interfaces, the oxygen ions at the $\mathrm{TiO}_{2}(\mathrm{R})$ surface are more difficult to diffuse outward to oxidize the reactive plasma species absorbed on the substrate surface, therefore, results in the less concentration of oxygen vacancies.

\section{CONCLUSION}

To conclude, we have successfully demonstrated the formation of 2DEG at the interface between a-LAO and the $\mathrm{TiO}_{2}(\mathrm{~A})$ as well as $\mathrm{TiO}_{2}(\mathrm{R})$ substrates. The $\mathrm{TiO}_{2}(\mathrm{~A})$-based heterostructure presents a comparable carrier density to the intensively-investigated system of a-LAO/STO, but with a much lower mobility even at low temperatures due to the occurrence of Kondo effect. In contrast, the a$\mathrm{LAO} / \mathrm{TiO}_{2}(\mathrm{R})$ gives rise to a low carrier density which is more than two orders of magnitude lower than that of a-LAO/STO and a-LAO/ $\mathrm{TiO}_{2}(\mathrm{~A})$ at low temperatures. The origin of the interfacial $2 \mathrm{DEGs}$ is further investigated by XPS. The lower carrier density of a- $\mathrm{LAO} / \mathrm{TiO}_{2}(\mathrm{R})$ is probably due to its larger activation energy for the diffusion of oxygen vacancy at the $\mathrm{TiO}_{2}(\mathrm{R})$ surface than the other two 
heterostructures. This work demonstrates the possibility to tune the conductive oxide heterointerface by changing the chemical environment.

\section{Supporting Information}

The Supporting Information is available from the ACS Publications or from the author.

XRD data of $\mathrm{TiO}_{2}(\mathrm{~A})$ substrate; $\mathrm{AFM}$ images of $\mathrm{STO}, \mathrm{TiO}_{2}(\mathrm{~A})$ and $\mathrm{TiO}_{2}(\mathrm{R})$ substrates; Twoband model fitting for the nonlinear Hall resistance of a-LAO/STO heterointerface; 2D weak localization fitting for the $R_{\mathrm{S}}$ of a-LAO/TiO $(\mathrm{A})$ heterointerface; The transport information for a$\mathrm{LAO} / \mathrm{STO}, \mathrm{a}-\mathrm{LAO} / \mathrm{TiO}_{2}(\mathrm{~A})$ and a-LAO/TiO $2(\mathrm{R})$ with different thickness $(3 \mathrm{~nm}, 16 \mathrm{~nm})$ of capping layers.

\section{AUTHOR INFORMATION}

\section{Corresponding Author:}

*E-mail: yunc@dtu.dk*

jrsun@iphy.ac.cn*

\section{ACKNOWLEDGEMENT}

This work has been supported by the National Basis Research of China (2013CB921700, 2016YFA0300701), the National Natural Science Foundation of China (11520101002, 51590880, 11374348, 11134007, 11574376 and 11574366) and the Key Program of the Chinese Academy of Sciences. 


\section{REFERENCE:}

(1) Ohtomo, A.; Hwang, H. Y.; A High-Mobility Electron Gas at the $\mathrm{LaAlO}_{3} / \mathrm{SrTiO}_{3}$ Heterointerface. Nature 2004, 427, 423-426.

(2) Chen, Y. Z.; Trier, F.; Kasama, T.; Christensen, D. V.; Bovet, N.; Balogh, Z. I.; Li, H.; Thyden, K. T.; Zhang, W.; Yazdi, S.; Norby, P.; Pryds, N.; Linderoth, S.; Creation of High Mobility TwoDimensional Electron Gases via Strain Induced Polarization at an otherwise Nonpolar Complex Oxide Interface. Nano Lett. 2015, 15, 1849-1854.

(3) Perna, P.; Maccariello, D.; Radovic, M.; Scotti di Uccio, U.; Pallecchi, I.; Codda, M.; Marré, D.;

Cantoni, C.; Gazquez, J.; Varela, M.; Pennycook, S. J.; Granozio, F. M.; Conducting Interfaces between Band Insulating Oxides: The $\mathrm{LaGaO}_{3} / \mathrm{SrTiO}_{3}$ Heterostructure. Appl. Phys. Lett. 2010, 97, 152111.

(4) Chen, Y. Z.; Bovet, N.; Trier, F.; Christensen, D. V.; Qu, F. M.; Andersen, N. H.; Kasama, T.; Zhang, W.; Giraud, R.; Dufouleur, J.; Jespersen, T. S.; Sun, J. R.; Smith, A.; Nygard, J.; Lu, L.; Buchner, B.; Shen, B. G.; Linderoth, S.; Pryds, N.; A High-Mobility Two-Dimensional Electron Gas at the Spinel/Perovskite Interface of $\mathrm{Gamma}_{-} \mathrm{Al}_{2} \mathrm{O}_{3} / \mathrm{SrTiO}_{3}$. Nat. Commun. 2013, 4, 1371.

(5) Reyren, N.; Thiel, S.; Caviglia, A. D.; Kourkoutis, L. F.; Hammerl, G.; Richter, C.; Schneider, C. W.; Kopp, T.; Rüetschi, A. S.; Jaccard, D.; Gabay, M.; Muller, D. A.; Triscone, J. M.; Mannhart, J.; Superconducting Interfaces Between Insulating Oxides. Science 2007, 317, 1196-1199. 
(6) Brinkman, A.; Huijben, M.; van Zalk, M.; Huijben, J.; Zeitler, U.; Maan, J. C.; van der Wiel, W. G.; Rijnders, G.; Blank, D. H.; Hilgenkamp, H.; Magnetic Effects at the Interface Between Non-Magnetic Oxides. Nat. Mater. 2007, 6, 493-496.

(7) Lin, W. N.; Ding, J. F.; Wu, S. X.; Li, Y. F.; Lourembam, J.; Shannigrahi, S.; Wang, S. J.; Wu, T.; Electrostatic Modulation of $\mathrm{LaAlO}_{3} / \mathrm{SrTiO}_{3}$ Interface Transport in an Electric Double-Layer Transistor. Adv. Mater. Interfaces 2014, 1, 1300001.

(8) Lei, Y.; Li, Y.; Chen, Y. Z.; Xie, Y. W.; Chen, Y. S.; Wang, S. H.; Wang, J.; Shen, B. G.; Pryds, N.; Hwang, H. Y.; Sun, J. R.; Visible-Light-Enhanced Gating Effect at the $\mathrm{LaAlO}_{3} / \mathrm{SrTiO}_{3}$ Interface. Nat. Commun. 2014, 5, 5554.

(9) Chen, Y. Z.; Green, R. J.; Sutarto, R.; He, F. Z.; Linderoth, S.; Sawatzky, G. A.; Pryds, N.; Tuning the Two-Dimensional Electron Liquid at Oxide Interfaces by Buffer-Layer-Engineered Redox Reactions. Nano Lett. 2017, 17, 7062-7066.

(10) Chen, Y. Z.; Trier, F.; Wijnands, T.; Green, R. J.; Gauquelin, N.; Egoavil, R.; Christensen, D. V.; Koster, G.; Huijben, M.; Bovet, N.; Macke, S.; He, F.; Sutarto, R.; Andersen, N. H.; Sulpizio, J. A.; Honig, M.; Prawiroatmodjo, G. E.; Jespersen, T. S.; Linderoth, S.; Ilani, S.; Verbeeck, J.; Van Tendeloo, G.; Rijnders, G.; Sawatzky, G. A.; Pryds, N.; Extreme Mobility Enhancement of TwoDimensional Electron Gases at Oxide Interfaces by Charge-Transfer-Induced Modulation Doping. Nat. Mater. 2015, 14, 801-806.

(11) Wu, S.; Luo, X.; Turner, S.; Peng, H.; Lin, W.; Ding, J.; David, A.; Wang, B.; Van Tendeloo, G.; Wang, J.; Wu, T.; Nonvolatile Resistive Switching in $\mathrm{Pt} / \mathrm{LaAlO}_{3} / \mathrm{SrTiO}_{3}$ Heterostructures. Phys. Rev. $X$ 2013, 3, 041027. 
(12) Nakagawa, N.; Hwang, H. Y.; Muller, D. A.; Why Some Interfaces Cannot be Sharp. Nat. Mater. 2006, 5, 204-209.

(13) Siemons, W.; Koster, G.; Yamamoto, H.; Harrison, W. A.; Lucovsky, G.; Geballe, T. H.; Blank, D. H.; Beasley, M. R.; Origin of Charge Density at $\mathrm{LaAlO}_{3}$ on $\mathrm{SrTiO}_{3}$ Heterointerfaces: Possibility of Intrinsic Doping. Phys. Rev. Lett. 2007, 98, 196802.

(14) Herranz, G.; Basletic, M.; Bibes, M.; Carretero, C.; Tafra, E.; Jacquet, E.; Bouzehouane, K.; Deranlot, C.; Hamzic, A.; Broto, J. M.; Barthelemy, A.; Fert, A.; High Mobility in $\mathrm{LaAlO}_{3} / \mathrm{SrTiO}_{3}$ Heterostructures: Origin, Dimensionality, and Perspectives. Phys. Rev. Lett. 2007, 98, 216803.

(15) Kalabukhov, A.; Gunnarsson, R.; Börjesson, J.; Olsson, E.; Claeson, T.; Winkler, D.; Effect of Oxygen Vacancies in the $\mathrm{SrTiO}_{3}$ Substrate on the Electrical Properties of the $\mathrm{LaAlO}_{3} / \mathrm{SrTiO}_{3}$ Interface. Phys. Rev. B 2007, 75, 121404.

(16) Chambers, S. A.; Engelhard, M. H.; Shutthanandan, V.; Zhu, Z.; Droubay, T. C.; Qiao, L.; Sushko, P. V.; Feng, T.; Lee, H. D.; Gustafsson, T.; Garfunkel, E.; Shah, A. B.; Zuo, J. M.; Ramasse, Q. M.; Instability, Intermixing and Electronic Structure at the Epitaxial $\mathrm{LaAlO}_{3} / \mathrm{SrTiO}_{3}(001)$ Heterojunction. Surf. Sci. Rep. 2010, 65, 317-352.

(17) Gunkel, F.; Hoffmann-Eifert, S.; Heinen, R. A.; Christensen, D. V.; Chen, Y. Z.; Pryds, N.; Waser, R.; Dittmann, R.; Thermodynamic Ground States of Complex Oxide Heterointerfaces. ACS Appl. Mater. Interfaces 2017, 9, 1086-1092. 
(18) Chen, Y. Z.; Pryds, N.; Kleibeuker, J. E.; Koster, G.; Sun, J.; Stamate, E.; Shen, B.; Rijnders, G.; Linderoth, S.; Metallic and Insulating Interfaces of Amorphous $\mathrm{SrTiO}_{3}$-based Oxide Heterostructures. Nano Lett. 2011, 11, 3774-3778.

(19) Chen, Y. Z.; Bovet, N.; Kasama, T.; Gao, W. W.; Yazdi, S.; Ma, C.; Pryds, N.; Linderoth, S.; Room Temperature Formation of High-Mobility Two-Dimensional Electron Gases at Crystalline Complex Oxide Interfaces. Adv. Mater. 2014, 26, 1462-1467.

(20) Shibuya, K.; Ohnishi, T.; Lippmaa, M.; Oshima, M.; Metallic Conductivity at the $\mathrm{CaHfO}_{3}$ / $\mathrm{SrTiO}_{3}$ Interface. Appl. Phys. Lett. 2007, 91, 232106.

(21) Posadas, A. B.; Kormondy, K. J.; Guo, W.; Ponath, P.; Geler-Kremer, J.; Hadamek, T.; Demkov, A. A.; Scavenging of Oxygen from $\mathrm{SrTiO}_{3}$ During Oxide Thin Film Deposition and the Formation of Interfacial 2DEGs. J. Appl. Phys. 2017, 121, 105302.

(22) Chen, Y. Z.; Christensen, D. V.; Trier, F.; Pryds, N.; Smith, A.; Linderoth, S.; On the Origin of Metallic Conductivity at the Interface of $\mathrm{LaAlO}_{3} / \mathrm{SrTiO}_{3}$. Appl. Surf. Sci. 2012, 258, 9242-9245.

(23) Lee, S. W.; Liu, Y.; Heo, J.; Gordon, R. G.; Creation and Control of Two-Dimensional Electron Gas Using Al-Based Amorphous Oxides/SrTiO 3 Heterostructures Grown by Atomic Layer Deposition. Nano Lett. 2012, 12, 4775-4783.

(24) Rodel, T. C.; Fortuna, F.; Sengupta, S.; Frantzeskakis, E.; Le Fevre, P.; Bertran, F.; Mercey, B.; Matzen, S.; Agnus, G.; Maroutian, T.; Lecoeur, P.; Santander-Syro, A. F.; Universal Fabrication of 2D Electron Systems in Functional Oxides. Adv. Mater. 2016, 28, 1976-1980. 
(25) Chen, Y. Z.; Döbeli, M.; Pomjakushina, E.; Gan, Y. L.; Pryds, N.; Lippert, T.; Scavenging of Oxygen Vacancies at Modulation-Doped Oxide Interfaces: Evidence from Oxygen Isotope Tracing. Phys. Rev. Mater. 2017, 1, 052002.

(26) Schneider, C. W.; Esposito, M.; Marozau, I.; Conder, K.; Doebeli, M.; Hu, Y.; Mallepell, M.; Wokaun, A.; Lippert, T.; The Origin of Oxygen in Oxide Thin Films: Role of the Substrate. Appl. Phys. Lett. 2010, 97, 192107.

(27) Scigaj, M.; Gázquez, J.; Varela, M.; Fontcuberta, J.; Herranz, G.; Sánchez, F.; Conducting Interfaces Between Amorphous Oxide Layers and $\mathrm{SrTiO}_{3}(110)$ and $\mathrm{SrTiO}_{3}$ (111). Solid State Ionics 2015, 281, 68-72.

(28) Sarkar, T.; Gopinadhan, K.; Zhou, J.; Saha, S.; Coey, J. M.; Feng, Y. P.; Ariando; Venkatesan, T.; Electron Transport at the $\mathrm{TiO}_{2}$ Surfaces of Rutile, Anatase, and Strontium Titanate: The Influence of Orbital Corrugation. ACS Appl. Mater. Interfaces 2015, 7, 24616-24621.

(29) Cheng, J.; Luo, J.; Yang, K.; Comparison Studies of Interfacial Electronic and Energetic Properties of $\mathrm{LaAlO}_{3} / \mathrm{TiO}_{2}$ and $\mathrm{TiO}_{2} / \mathrm{LaAlO}_{3}$ Heterostructures from First-Principles Calculations. ACS Appl. Mater. Interfaces 2017, 9, 7682-7690.

(30) Lechermann, F.; Heckel, W.; Kristanovski, O.; Müller, S.; Oxygen-Vacancy Driven Electron Localization and Itinerancy in Rutile-Based $\mathrm{TiO}_{2}$. Phys. Rev. B 2017, 95, 195159.

(31) Kim, J. S.; Seo, S. S. A.; Chisholm, M. F.; Kremer, R. K.; Habermeier, H. U.; Keimer, B.; Lee, H. N.; Nonlinear Hall Effect and Multichannel Conduction in $\mathrm{LaTiO}_{3} / \mathrm{SrTiO}_{3}$ Superlattices. Phys. Rev. B 2010, 82, 201407. 
(32) Zhang, S.; Ogale, S. B.; Yu, W.; Gao, X.; Liu, T.; Ghosh, S.; Das, G. P.; Wee, A. T. S.; Greene, R. L.; Venkatesan, T.; Electronic Manifestation of Cation-Vacancy-Induced Magnetic Moments in a Transparent Oxide Semiconductor: Anatase Nb:TiO 2. Adv. Mater. 2009, 21, 2282-2287.

(33) Jun, K.; Resistance Minimum in Dilute Magnetic Alloys. Progr. Theor. Phys. 1964, 32, 37-49.

(34) Li, Y.; Deng, R.; Lin, W.; Tian, Y.; Peng, H.; Yi, J.; Yao, B.; Wu, T.; Electrostatic Tuning of Kondo Effect in a Rare-Earth-Doped Wide-Band-Gap Oxide. Phys. Rev. B 2013, 87, 155151.

(35) Yan, H.; Zhang, Z.; Wang, S.; Zhang, H.; Chen, C. L.; Jin, K.; Modulated Transport Behavior of Two-Dimensional Electron Gas at Ni-Doped $\mathrm{LaAlO}_{3} / \mathrm{SrTiO}_{3}$ Heterointerfaces. ACS Appl. Mater. Interfaces 2017, 9, 39011-39017.

(36) Lee, P. A.; Ramakrishnan, T. V.; Disordered Electronic Systems. Rev. Mod. Phys. 1985, 57, 287337.

(37) Lee, M.; Williams, J. R.; Zhang, S.; Frisbie, C. D.; Goldhaber-Gordon, D.; Electrolyte GateControlled Kondo Effect in $\mathrm{SrTiO}_{3}$. Phys. Rev. Lett. 2011, 107, 256601.

(38) Niu, W.; Gao, M.; Wang, X.; Song, F.; Du, J.; Wang, X.; Xu, Y.; Zhang, R.; Evidence of Weak Localization in Quantum Interference Effects Observed in Epitaxial $\mathrm{La}_{0.7} \mathrm{Sr}_{0.3} \mathrm{MnO}_{3}$ Ultrathin Films. Sci. Rep. 2016, 6, 26081.

(39) T. A. Costi; A. C. Hewson; Zlatic, V.; Transport Coefficients of the Anderson Model via the Numerical Renormalization Group. J. Phys.: Condens. Matter 1994, 6, 2519-2558.

(40) Goldhaber-Gordon, D.; Gores, J.; Kastner, M. A.; From the Kondo Regime to the Mixed-Valence Regime in a Single-Electron Transistor. Phys. Rev. Lett. 1998, 81, 5225-5228. 
(41) Zhang, G.; Gu, Q.; Kondo Spin Liquid and Magnetically Long-Range Ordered States in the Kondo Necklace Model. Phys. Rev. B 2000, 62, 69-72.

(42) Khan, W.; Naqvi, A. H.; Gupta, M.; Husain, S.; Kumar, R.; Small Polaron Hopping Conduction Mechanism in Fe Doped $\mathrm{LaMnO}_{3}$. J. Chem. Phys. 2011, 135, 054501.

(43) Jaćimović, J.; Horváth, E.; Náfrádi, B.; Gaál, R.; Nikseresht, N.; Berger, H.; Forró, L.; Magrez, A.; From Nanotubes to Single Crystals: Co doped $\mathrm{TiO}_{2}$. APL Mater. 2013, 1, 032111.

(44) Yildiz, A.; Lisesivdin, S. B.; Kasap, M.; Mardare, D.; Non-adiabatic Small Polaron Hopping Conduction in Nb-Doped $\mathrm{TiO}_{2}$ Thin Film. Physica B: Condensed Matter 2009, 404, 1423-1426.

(45) Wendt, S.; Sprunger, P. T.; Lira, E.; Madsen, G. K. H.; Li, Z. S.; Hansen, J. Ø.; Matthiesen, J.; Blekinge-Rasmussen, A.; Lægsgaard, E.; Hammer, B.; Besenbacher, F.; The Role of Interstitial Sites in the Ti3d Defect State in the Band Gap of Titania. Science 2008, 320, 1755-1759.

(46) Yim, C. M.; Pang, C. L.; Thornton, G.; Oxygen Vacancy Origin of the Surface Band-Gap State of $\mathrm{TiO}_{2}$ (110). Phys. Rev. Lett. 2010, 104, 036806.

(47) Carrasco, J.; Illas, F.; Lopez, N.; Kotomin, E. A.; Zhukovskii, Y. F.; Evarestov, R. A.; Mastrikov, Y. A.; Piskunov, S.; Maier, J.; First-Principles Calculations of the Atomic and Electronic Structure of F Centers in the Bulk and on the (001) Surface of $\mathrm{SrTiO}_{3}$. Phys. Rev. B 2006, 73, 064106.

(48) Linh, N. H.; Nguyen, T. Q.; Diño, W. A.; Kasai, H.; Effect of Oxygen Vacancy on the Adsorption of $\mathrm{O}_{2}$ on Anatase $\mathrm{TiO}_{2}$ (001): A DFT-Based Study. Surf. Sci. 2015, 633, 38-45. 
(49) Benjamin, J. M.; Graeme, W. W.; A Density Functional Theory + U Study of Oxygen Vacancy Formation at the (110), (100), (101), and (001) Surfaces of Rutile $\mathrm{TiO}_{2}$. J. Phys. Chem. C 2009, 113, $7322-7328$.

(50) Uberuaga, B. P.; Bai, X. M.; Defects in Rutile and Anatase Polymorphs of TiO2: Kinetics and Thermodynamics near Grain Boundaries. J. Phys: Condens. Matter. 2011, 23, 435004. 


\section{Figures and Figure captions:}

FIG. 1 (a) A schematic illustration of the oxide heterostructure, consisting of an amorphous $\mathrm{LaAlO}_{3}$ (aLAO) capping layer and a $\mathrm{TiO}_{2}$-based substrate. The unit cell lattice and the Ti-O configuration (Ti-OTi bond angle) of the three types of substrates: (b) $\mathrm{SrTiO}_{3}(\mathrm{STO})$, (c) anatase $\mathrm{TiO}_{2}\left[\mathrm{TiO}_{2}(\mathrm{~A})\right]$ and (d) rutile $\mathrm{TiO}_{2}\left[\mathrm{TiO}_{2}(\mathrm{R})\right]$.

FIG. 2 Temperature dependence of (a) sheet resistance, $R_{\mathrm{s}}$, (b) carrier density, $n_{\mathrm{s}}$, and (c) electron Hall mobility, $\mu$, of 2DEGs created at the interface between the capping layer of a-LAO and the substrate of $\mathrm{TiO}_{2}$-terminated $\mathrm{STO}$ (blue circles), $\mathrm{TiO}_{2}(\mathrm{~A})$ (green squares) and $\mathrm{TiO}_{2}(\mathrm{R})$ (purple triangles), respectively.

FIG. 3 (a) Magnetic field dependence of the Hall resistance $\left(R_{\mathrm{xy}}\right)$ for the a-LAO/STO interface, measured in the temperature from $295 \mathrm{~K}$ to $2 \mathrm{~K}$. (b) Temperature-dependent $R_{\mathrm{S}}$ of the a-LAO/TiO $2(\mathrm{~A})$ (green dots) is fitted by Kondo effect (black line). (c) Temperature-dependent $\ln \left(R_{\mathrm{S}} / T\right)$ of the a$\mathrm{LAO} / \mathrm{TiO}_{2}(\mathrm{R})$ (purple dots) is fitted by small polaron model (black line).

FIG. 4 (a) The Ti $2 p$ X-ray photoelectron spectroscopy (XPS) of a-LAO/STO, a-LAO/TiO $2(A)$ and a$\mathrm{LAO} / \mathrm{TiO}_{2}(\mathrm{R})$. (b)-(d) Fitting of the XPS intensity signals of these three heterostructures. The measurements are performed at room temperature. 
FIG. 1

(a)

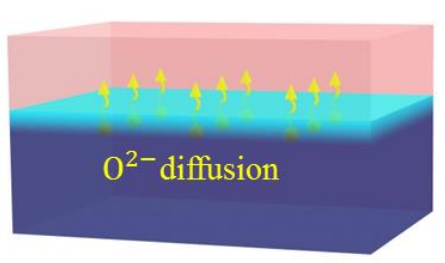

a-LAO

2DEG

Substrate

(b) STO

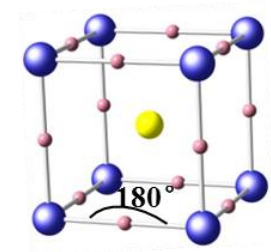

$a=b=c=3.905 \AA$

OTiC sreO (c) $\mathrm{TiO}_{2}(\mathrm{~A})$

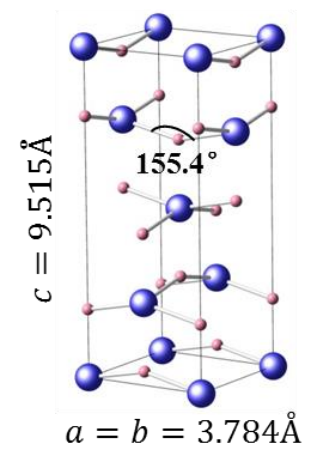

(d) $\quad \mathrm{TiO}_{2}(\mathrm{R})$

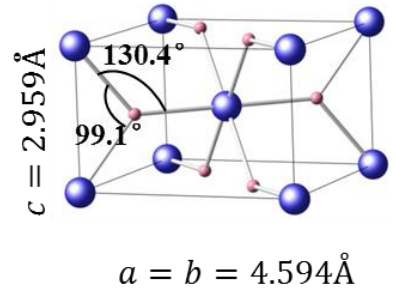


FIG. 2
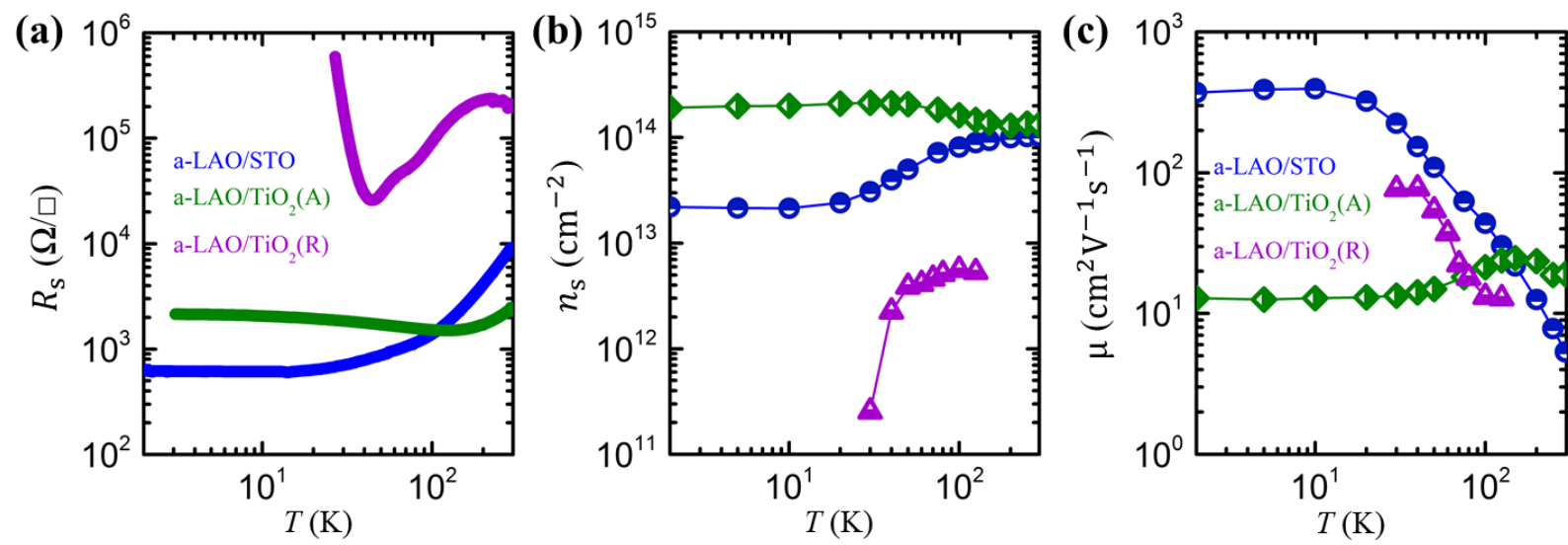
FIG. 3
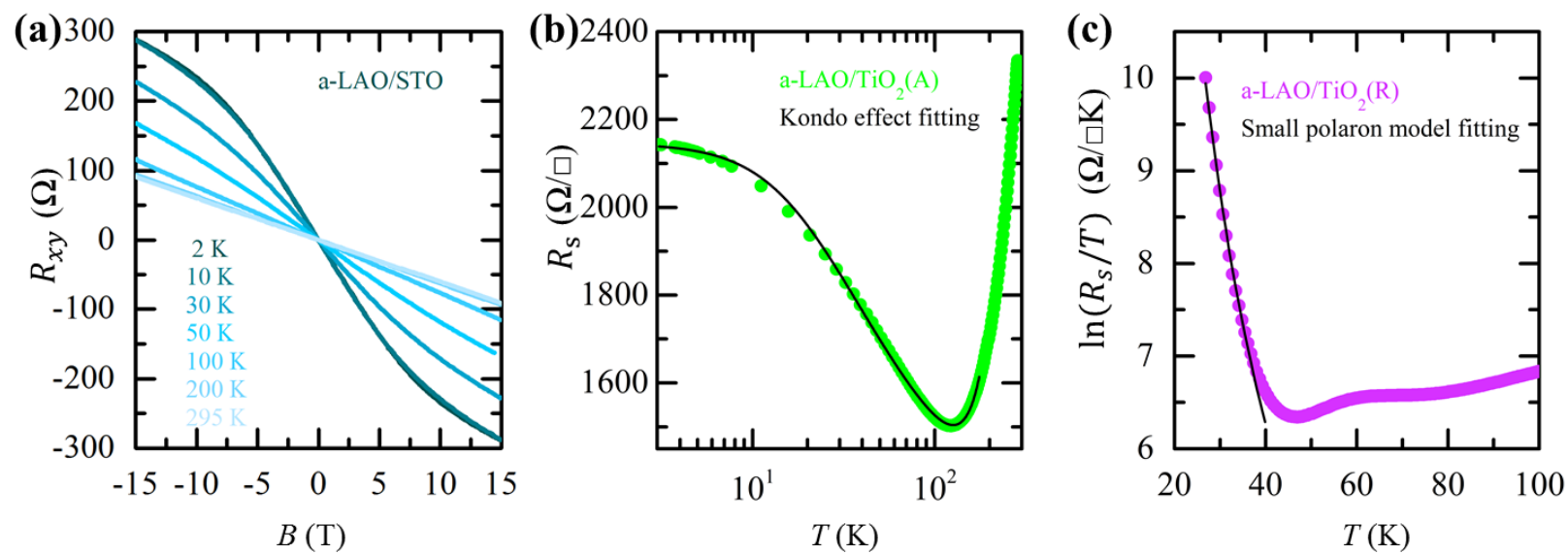
FIG. 4
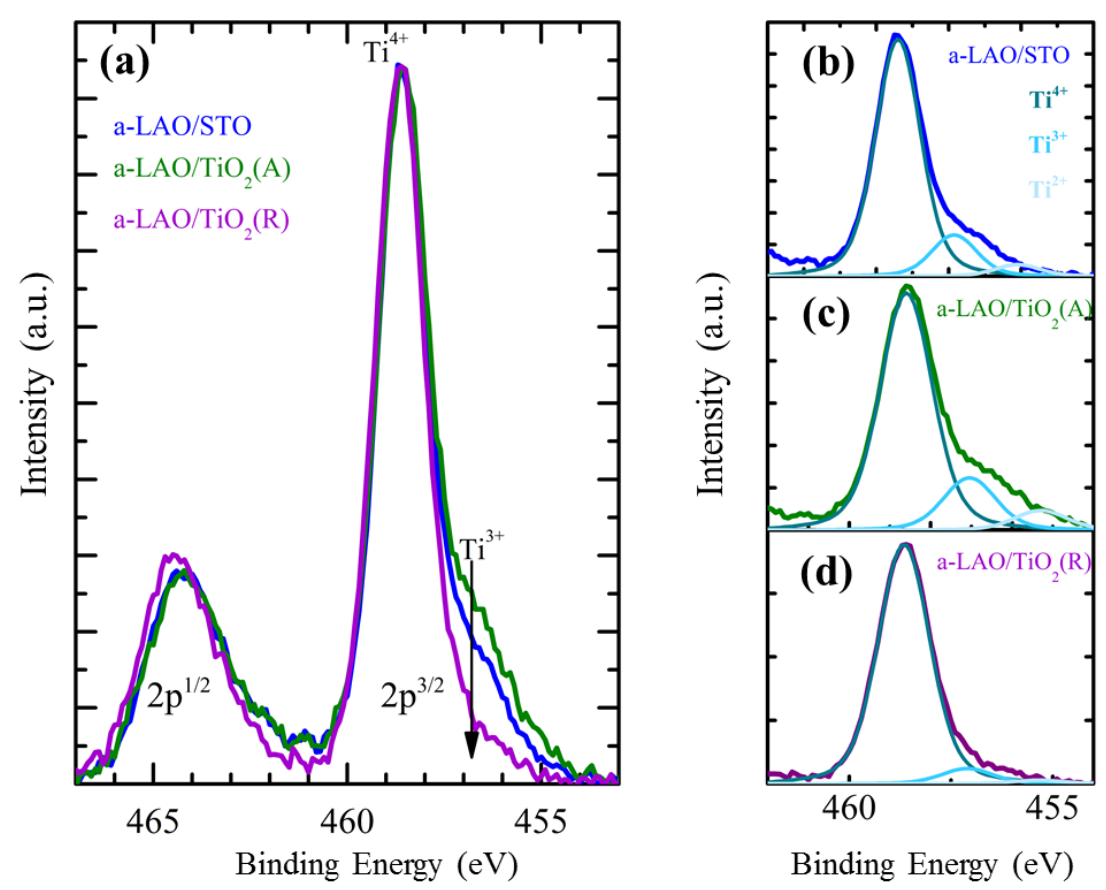
Table I. The amounts of cations determined by XPS measurements and the Hall carrier densities at 295 $\mathrm{K}$ in the three heterointerfaces:

\begin{tabular}{|c|c|c|c|c|}
\hline & $\mathrm{Ti}^{4+}(\%)$ & $\mathrm{Ti}^{3+}(\%)$ & $\mathrm{Ti}^{2+}(\%)$ & $n_{\mathrm{s}}\left(\mathrm{cm}^{-2}\right)$ \\
\hline a-LAO/STO & 79.4 & 15.9 & 4.7 & $1.0 \times 10^{14}$ \\
\hline $\mathrm{a}-\mathrm{LAO} / \mathrm{TiO}_{2}(\mathrm{~A})$ & 76.0 & 17.5 & 6.5 & $1.3 \times 10^{14}$ \\
\hline $\mathrm{a}-\mathrm{LAO} / \mathrm{TiO}_{2}(\mathrm{R})$ & 92.5 & 5.7 & 1.8 & $5.6 \times 10^{12}$ \\
\hline
\end{tabular}

Table II. The formation energy of an oxygen vacancy and the activation energy for the diffusion of oxygen vacancy on the surface of $\mathrm{STO}, \mathrm{TiO}_{2}(\mathrm{~A})$ and $\mathrm{TiO}_{2}(\mathrm{R})$ :

\begin{tabular}{llll}
\hline & $\mathrm{STO}$ & $\mathrm{TiO}_{2}(\mathrm{~A})$ & $\mathrm{TiO}_{2}(\mathrm{R})$ \\
\hline Formation energy (eV) & $5.94^{47}$ & $4.00-4.96^{48}$ & $3.21^{49}$ \\
Activation energy (eV) & $0.1-0.2^{47}$ & $0.2^{50}$ & $1.5^{50}$ \\
\hline
\end{tabular}


Table of Contents Graphic

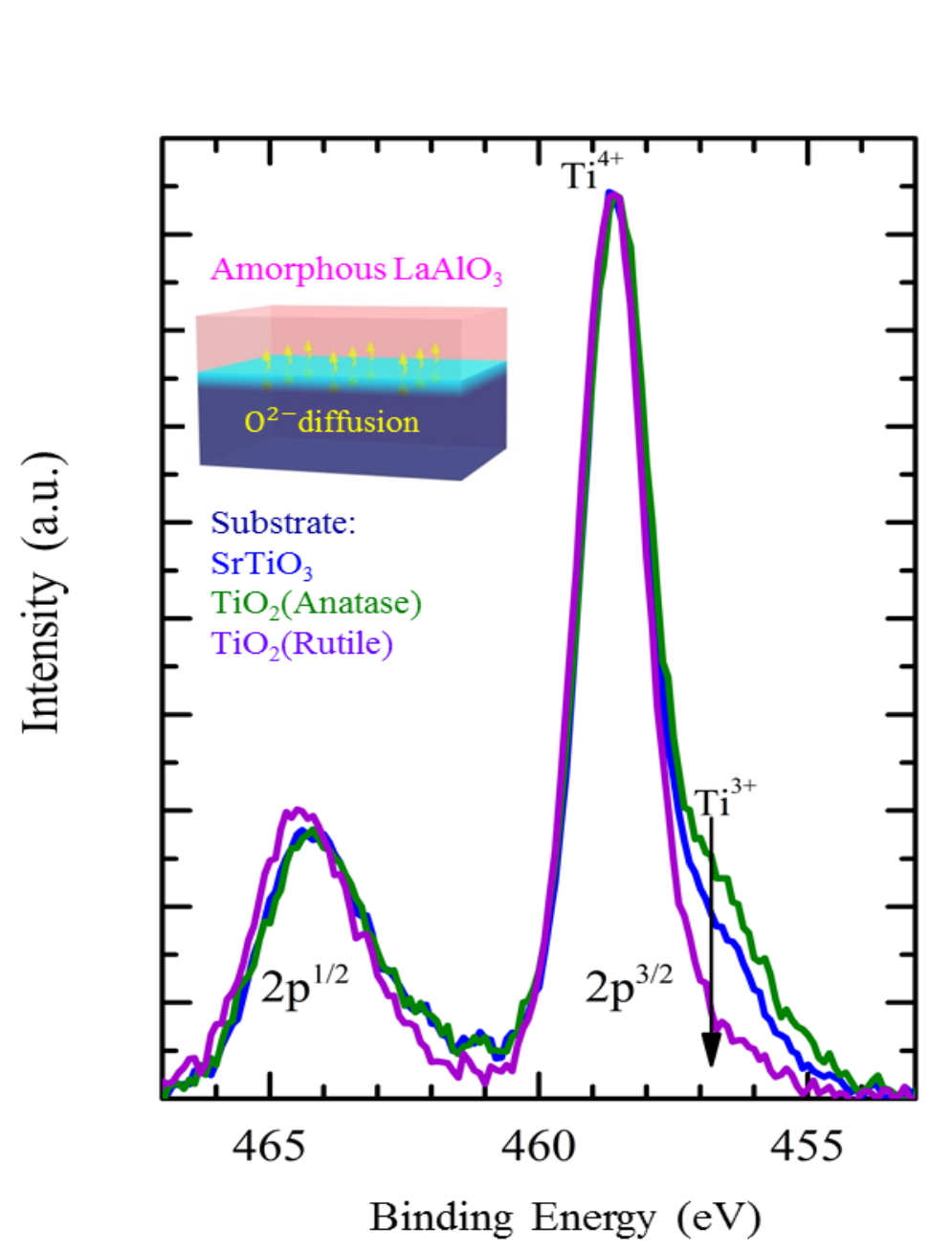

